\title{
Highly Efficient Coaxial TiO2- PtPd Tubular Nanomachines for Photocatalytic Water Purification with Multiple Locomotion Strategies
}

\section{Journal Article}

Author(s):

Musthaq, Fajer; Asani, Agim; Hoop, Marcus; Chen, Xiang-Zhong; Ahmed, Daniel; Nelson, Bradley J.; Pané, Salvador

Publication date:

2016-10

Permanent link:

https://doi.org/10.3929/ethz-b-000118858

Rights / license:

In Copyright - Non-Commercial Use Permitted

Originally published in:

Advanced Functional Materials 26(38), https://doi.org/10.1002/adfm.201602315 
Article type: Full Paper

Highly Efficient Coaxial TiO2-PtPd Tubular Nanomachines for Photocatalytic Water Purification with Multiple Locomotion Strategies

By Fajer Mushtaq, Agim Asani, Marcus Hoop, Xiang-Zhong Chen, * Daniel Ahmed, Bradley J. Nelson, and Salvador Pané*

F. Mushtaq, A. Asani, M. Hoop, Dr. X.-Z. Chen, Dr. D. Ahmed, Prof. Dr. B. J. Nelson, Dr. S. Pané

Multi-Scale Robotics Lab (MSRL), Institute of Robotics and Intelligent Systems (IRIS), ETH Zurich, CH-8092 Zurich, Switzerland.

E-mail: chenxian@ethz.ch, vidalp@ethz.ch

Keywords: $\mathrm{TiO}_{2}$; Visible light photocatalysis; Core-shell nanotubes; Electrochemical synthesis; Micromanipulation 


\begin{abstract}
Titania is a promising photocatalyst for water purification or production of solar fuels. However, due to its large bandgap, titania is photoactive solely under UV light, which accounts for less than $5 \%$ of the solar spectrum. In this work, we have designed and fabricated $\mathrm{TiO}_{2}$-based hybrid one-dimensional nanostructures with photocatalytic activities extended to visible light region. Highly efficient coaxial $\mathrm{TiO}_{2}-\mathrm{PtPd}-\mathrm{Ni}$ nanotubes were fabricated by a template-assisted electrochemical synthesis route for water remediation under UV light, visible light and natural sunlight. These coaxial hybrid nanotubes displayed a 100\% degradation of organic pollutant rhodamine B in only $50 \mathrm{~min}\left(k\right.$-value $\left.0.071 \mathrm{~min}^{-1}\right)$ and 30 min under visible light and natural sunlight, respectively. For comparison, $\mathrm{TiO}_{2}$ nanotubes doped with Pd nanoparticles were also fabricated and they showed inferior photocatalytic properties and degrading stability over time. The multicomponent design enabled us to actuate the hybrid NTs by using two different external energy sources i.e. magnetic and acoustic fields. Self-propelled, autonomous actuation in the presence of $\mathrm{H}_{2} \mathrm{O}_{2}$ was also realized. These versatile actuation modes have the potential to enable the reported photocatalytic nanomachines to work efficiently under complex environments and to be easily collected for re-use.
\end{abstract}




\section{Introduction}

Environmental pollution caused by increasing industrialization is one of the major problems imposed on modern society. ${ }^{[1]}$ It is suggested that more than 14,000 deaths are reported daily due to waterborne diseases. ${ }^{[2]}$ Industry is the biggest polluter of water bodies, with the textile industry discharging more than 200,000 tons of toxic synthetic dyes every year into effluents due to inefficiencies in the dyeing process. ${ }^{[3]}$ The presence of even trace amounts of such synthetic dyes in water is considered harmful due to their high chemical oxygen demand (COD) and carcinogenic nature. ${ }^{[2 a, 4]}$ Pollutants are also responsible for negatively altering aquatic ecosystems by blocking the penetration of sunlight and oxygen, essential for the survival of various aquatic forms. ${ }^{[5]}$ Unfortunately, due to their high stability, most of these recalcitrant dyes escape conventional wastewater treatment processes such as pre-chlorination, flocculation, coagulation and flotation and persist in the environment. ${ }^{[1,6]}$ Consequently, there is an ever-increasing need to develop efficient, cost-effective and green techniques for water purification. The heterogeneous advanced oxidation process (AOP) is one of the most extensively studied approaches, where toxic organic dyes are completely degraded into harmless by-products such as $\mathrm{CO}_{2}$ and $\mathrm{H}_{2} \mathrm{O}$. AOPs are based on the in-situ generation of highly reactive species, such as hydroxyl and superoxide anion radicals $\left(\mathrm{OH}^{\bullet}\right.$ and $\mathrm{O}_{2}{ }^{*-}$, respectively), by semiconductor photocatalysts for degradation of organic compounds. ${ }^{[7]}$

Due to its large band-gap, nontoxicity, low cost and abundant availability, titanium dioxide $\left(\mathrm{TiO}_{2}\right)$ is one of the most extensively studied semiconductors for photocatalysis. ${ }^{[8]} \mathrm{A}$ wide band-gap provides sufficient charge separation between the photogenerated electronhole pairs, hence retarding their recombination and increasing the efficiency of the system. ${ }^{[9]}$ However, this wide band-gap also limits the use of $\mathrm{TiO}_{2}$ to only UV light, which accounts for less than $5 \%$ of the solar spectrum. ${ }^{[10]}$ To extend $\mathrm{TiO}_{2}$ 's optical response into visible light, many attempts have been explored to modify its band gap. These measures include doping and coupling $\mathrm{TiO}_{2}$ with nonmetals (e.g. sulfur, carbon, nitrogen), semiconductors, and 
metals. ${ }^{[11]}$ Among these techniques, fabrication of heterostructures of $\mathrm{TiO}_{2}$ with noble metals such as gold $(\mathrm{Au})$, silver $(\mathrm{Ag})$, platinum $(\mathrm{Pt})$ or palladium $(\mathrm{Pd})$ has proven to be one of the most efficient approaches. ${ }^{[12]}$ The combination of an n-type semiconductor like $\mathrm{TiO}_{2}$ with a noble metal creates a Schottky barrier at their interface. This Schottky barrier is responsible for enhancing electron-hole charge separation and hence, is responsible for prolonging the lifetime of these charge carriers. ${ }^{[13]}$ Moreover, when $\mathrm{TiO}_{2}$ is coupled with bimetallic noble metals such as platinum-copper, silver-palladium or platinum-palladium, its photocatalytic activity is much higher. ${ }^{[14]}$ For example, $\mathrm{TiO}_{2}$ loaded with bimetallic platinum-palladium nanoparticles (NPs) exhibited a photocatalytic activity that was 19 times higher than that of pure $\mathrm{TiO}_{2}$ and about approximately four times higher compared to coupling only monometallic Pd NPs to $\mathrm{TiO}_{2}{ }^{[14 \mathrm{a}]}$

Besides appropriate material selection, development of highly efficient photocatalysts also requires selection of their optimal size and architecture. Due to the very high specific surface area of one-dimensional nanowires (NWs) and nanotubes (NTs), they are ideal candidates to develop solar cells and photocatalysts for water remediation and water splitting. ${ }^{[15]}$ With a charge carrier diffusion length of about $20 \mathrm{~nm}, \mathrm{TiO}_{2}$ nanostructures with a thickness of less than $20 \mathrm{~nm}$ are optimal for providing efficient charge separation. ${ }^{[13 \mathrm{a}, 16]}$ This makes thin $\mathrm{TiO}_{2}$ NTs with a high aspect ratio an attractive choice to fabricate efficient photocatalysts. Two major approaches are commonly used to combine noble metals with $\mathrm{TiO}_{2}$, which includes either depositing NPs on the surface of $\mathrm{TiO}_{2}$ or fabricating core-shell noble metal- $\mathrm{TiO}_{2}$ nanospheres. ${ }^{[17]}$ Photocatalysts based on NPs deposited on a $\mathrm{TiO}_{2}$ surface have shown a reduced stability caused by dissolution or photocorrosion of NPs in their surrounding environment. ${ }^{[12 \mathrm{a}, 18]}$ In contrast, the architecture exhibited by encapsulation of noble metals around a $\mathrm{TiO}_{2}$ shell could lead to development of more efficient and durable photoactive materials in the future. ${ }^{[18-19]}$ Presently, various synthesis routes are available for fabrication of high specific surface area $\mathrm{TiO}_{2}$ nanostructures. ${ }^{[8 \mathrm{a}, 11 \mathrm{c}]}$ Unfortunately, these techniques lack 
control over the growth parameters and suffer from the inability to fabricate multicomponent nanostructures. However, electrochemical synthesis can be used to fabricate hybrid $\mathrm{TiO}_{2}$ nanostructures with a high degree of control over their morphology, composition, and design. [20]

Another important fact that prevents photocatalysts from being used for practical water purification applications is a lack of control over their motion and swimming characteristics. ${ }^{[21]}$ Most current micro- and nanostructures are designed to be propelled using a single power source such as external magnetic fields, electric fields, light, or by decomposition of chemical fuels such as hydrogen peroxide. ${ }^{[22]}$ This restriction causes secondary pollution due to their loss and renders them costly and ineffective under harsh, changing environments. Hence, there is a growing need to fabricate hybrid micro/nanomachines that are capable of achieving efficient propulsion by using multiple power sources. ${ }^{[23]}$ Achieving such a multimode propulsion strategy requires careful design and efficient implementation of a multicomponent nanovehicle within a single nanoscale entity.

In this work, we present nanomachines composed of coaxial $\mathrm{TiO}_{2}$-noble metal $(\mathrm{Pt}$, PtPd) NTs filled with nickel nanowires for photocatalytic water remediation under UV, visible light, and natural sunlight. An electrochemical synthesis route was chosen to fabricate these multicomponent nanostructures by sequential template-assisted electrodeposition. This approach provided a unique fabrication platform to study the enhancement in photocatalytic activity offered by noble metals coupled to $\mathrm{TiO}_{2}$ NTs as NPs and NTs. Comparison in the photocatalytic efficiency offered by bimetallic PtPd NTs versus monometallic Pd NTs was also investigated. Additionally, these hybrid NTs demonstrated versatile actuation modes such as fuel-powered autonomous actuation as well as fuel-free magnetic and acoustic field guided swimming behavior. 


\section{Results and discussion}

$\mathrm{TiO}_{2}$ nanotubes were deposited inside commercially available anodized aluminium oxide (AAO) templates by an electrochemically induced sol-gel technique (Figure 1a (i, ii)). When a potential greater than $-0.9 \mathrm{~V} v s . \mathrm{Ag} / \mathrm{AgCl}$ was applied, nitrate present in the solution was reduced to form hydroxyl ions causing a local increase in $\mathrm{pH}$ near the electrode. ${ }^{[24]}$ The $\mathrm{pH}$ increase induced formation of titanium hydroxide gel within the AAO pores. ${ }^{[16,24]}$ This gel was then thermally treated using two approaches in which AAO templates were either dried overnight at $100{ }^{\circ} \mathrm{C}\left(\mathrm{T}_{-} 100\right)$ to remove water or directly annealed at $650{ }^{\circ} \mathrm{C}$ for $2 \mathrm{~h}\left(\mathrm{~T}_{-} 650\right)$ to form crystalline $\mathrm{TiO}_{2} \mathrm{NTs}$. $\mathrm{TiO}_{2} \mathrm{NTs}$ dried at $100{ }^{\circ} \mathrm{C}$ were filled with Pd by electrodeposition (Figure 1a (iii)) and the NTs were eventually annealed at $650{ }^{\circ} \mathrm{C}$ in order to allow crystallization $\left(\mathrm{TP}_{-} 650\right)$. Apart from this, Pd and PtPd NTs were also electroplated inside the AAO templates that were previously filled with $\mathrm{TiO}_{2} \mathrm{NTs}$ annealed at $650{ }^{\circ} \mathrm{C}$ (Figure 1a (iv)) (T_650 P, T_650PP). These hybrid NTs were filled with Ni nanowires to efficiently collect them using a magnet. This was followed by wet etching of the AAO template to release the NTs (Figure 1a (v-vi)). Figure $1 \mathrm{~b}$ presents the scanning electron microscopy (SEM) image obtained from freestanding TP_650-Ni NTs, and the inset shows their magnified image. We can observe the presence of many randomly distributed NPs on the surface of $\mathrm{TiO}_{2} \mathrm{NTs}_{\text {. Figure }}$ 1c presents the high angle annular dark (HAADF) scanning transmission electron microscopy (STEM) image of a single TP_650 NT, where we can see its uniform NW structure. From the corresponding energy-dispersive X-ray (EDX) mappings obtained, we can clearly observe the presence of $\mathrm{a} \mathrm{TiO}_{2} \mathrm{NT}$ with randomly distributed Pd NPs encapsulating a Ni NW. The reason behind the formation of Pd NPs could be the inability of the titania gel to form dense shells due to the insufficient heat treatment, causing the Pd ions to penetrate the gel during electrodeposition. ${ }^{[16]}$ As a result, Pd nuclei formed when such ions combined with an electron and during the subsequent annealing step at $650{ }^{\circ} \mathrm{C}$, these $\mathrm{Pd}$ NPs diffused into the $\mathrm{TiO}_{2}$ phase. Figure $1 \mathrm{~d}$ shows the top-view of freestanding $\mathrm{T}_{-} 650 \mathrm{P}-\mathrm{Ni} \mathrm{NT}$ arrays and the inset shows their 
magnified image. A STEM image obtained for this sample is presented in Figure 1e, where a uniform shell can be seen around a nanowire. From this image, we can clearly observe that this hybrid nanostructure has conformal interfaces between its different components. According to the EDX mappings obtained for the elements, this outer shell clearly corresponds to a $15 \mathrm{~nm}$-thick $\mathrm{TiO}_{2}$ layer, followed by a Pd NT and Ni NW. Superimposed EDX map for $\mathrm{Ti}, \mathrm{O}$ and $\mathrm{Pd}$ elements further depicts a $\mathrm{TiO}_{2}$ shell homogeneously encapsulating a Pd NT.

The crystalline structure of the hybrid NTs was analyzed using TEM and X-ray diffraction (XRD) (Figure 2a). XRD investigation performed on these NTs showed that all samples possessed a good crystallinity, apart from the amorphous $T_{-} 100$ sample (data not shown). XRD investigation of $\mathrm{T}_{-} 650 \mathrm{NT}$ sample revealed that the $\mathrm{TiO}_{2}$ phase structure consists of a mixture of anatase, rutile and brookite polymorphs. The presence of all three polymorphs has been previously shown to have a positive effect on the photocatalytic activity of $\mathrm{TiO}_{2} \cdot{ }^{[25]}$ Analysis of the diffraction peaks at $25.4^{\circ}, 38.3^{\circ}$ and $75.3^{\circ}$ can be observed corresponding to the (101), (112) and (224) faces of anatase $\mathrm{TiO}_{2}$ (JCPDS file no. 73-1764). Similarly, 27.4, $54.8^{\circ}$ and $56.5^{\circ}$ peaks can be ascribed to (110), (211) and (220) crystal planes of rutile $\mathrm{TiO}_{2}$ (JCPDS file no. $73-1765$ ) and reflections at $31.7^{\circ}, 33.6^{\circ}, 42.0^{\circ}, 70.6^{\circ}$ and $71.6^{\circ}$ can be assigned to brookite $\mathrm{TiO}_{2}$ (JCPDS file no. 16-0617). Analysis of T_650P-Ni NTs showed new peaks at $40.1^{\circ}, 46.7^{\circ}$ and $68.2^{\circ}$ assigned to the (111), (200) and (220) faces of cubic Pd (JCPDS file no. 46-1043) and reflections at $44.6^{\circ}, 51.8^{\circ}, 64.6^{\circ}$ and $76.4^{\circ}$ corresponding to the (111), (200), (012) and (220) crystal planes of cubic Ni (JCPDS file no. 04-0850), respectively. XRD analysis of $\mathrm{T}_{-} 650 \mathrm{PP}-\mathrm{Ni}$ NTs also revealed new peaks at $38.9^{\circ}, 45.6^{\circ}$ and 66. $2^{\circ}$ that can be ascribed to the (111), (200) and (220) faces of cubic Pt (JCPDS file no. 040802).

A TEM image showing the top-end of two over-lapping T_650PP-Ni NTs is presented in Figure 2b. From this image, we can observe the presence of a thin shell, which encapsulates 
a NT with a dark contrast. The inset, shows the selected area electron diffraction (SAED) pattern of these NTs, where the presence of multiple rings confirms that the structures are polycrystalline in nature. High-resolution transmission electron microscopy (HRTEM) analysis performed on the outermost shell is presented in Figure 2c. From this, we can observe a $15 \mathrm{~nm}$-thick polycrystalline layer having multiple growth orientations. Further analysis shows the presence of d-values of $0.324 \mathrm{~nm}$ and $0.350 \mathrm{~nm}$, corresponding to $\mathrm{TiO}_{2}$ anatase (101) and rutile (110) planes. In contrast, by analyzing the HRTEM image obtained from the NT with a dark contrast (Figure 2d), presence of PtPd NT could be identified from the d-spacings of $0.196 \mathrm{~nm}$ and $0.224 \mathrm{~nm}(\mathrm{Pt}$ (200) and $\mathrm{Pd}(111)$, respectively).

The photocatalytic performance of all NTs was evaluated by studying the degradation of a rhodamine-B (RhB) solution under $\mathrm{UV}$, visible light, and natural sunlight for $1 \mathrm{~h}$ using continuous magnetic agitation. The degradation curves obtained under UV light show that all the samples exhibited a gradual degradation over time except the control that showed negligible response (Figure 3a). Due to their high crystallinity, all the samples that were annealed at a high temperature $\left(650{ }^{\circ} \mathrm{C}\right)$ showed a better performance than the ones dried at $100{ }^{\circ} \mathrm{C}\left(\mathrm{T}_{-} 100\right)$. The bimetallic $\mathrm{T}_{-} 650 \mathrm{PP}$ NTs were the most efficient photocatalysts with a 93\% dye degradation in $1 \mathrm{~h}$. From Figure $3 \mathrm{~b}$, we can observe that the trend of dye degradation of NTs in the presence of visible light is similar to their response under UV light, where all the NTs displayed a gradual degradation of RhB over time and the bimetallic samples were the best performing photocatalyst.

A quantitative analysis of the degradation rates offered by the high temperature annealed samples is given by comparing their reaction rate constant $k$, which can be defined by,

$$
k=\ln \left(\frac{C_{0}}{C}\right) / t
$$


where, $C_{o}$ is the initial $\mathrm{RhB}$ concentration and $C$ is the $\mathrm{RhB}$ concentration at time $t$. The calculation is based on the assumption that the kinetics of $\mathrm{RhB}$ degradation reaction catalyzed by these NTs are (pseudo-)first-order reactions. These results are presented in Figure 3c where we can observe that pure $\mathrm{TiO}_{2} \mathrm{~T}_{-} 650$ NTs showed a poorer performance under visible light than under UV light. Conversely, the hybrid NT samples coupled with noble metals (i.e. Pt or PtPd) performed better under visible light than under UV light. While hybrid NTs didn't show any significant improvement than the pure $\mathrm{TiO}_{2}$ NTs under UV light, coupling $\mathrm{TiO}_{2}$ nanotubes with $\mathrm{Pd}$ nanoparticles $\left(\mathrm{TP}_{-} 650\right), \mathrm{Pd}$ nanotubes $\left(\mathrm{T}_{650 \_} \mathrm{P}\right)$ and $\mathrm{PdPt}$ nanotubes ( $\mathrm{T}_{650 \_} \mathrm{PP}$ ) gives 3.2, 5.8, and 9.6 times higher $k$-values than pure $\mathrm{TiO}_{2}$ under visible light. The best performing photocatalysts were the T_650PP NTs that demonstrated $100 \%$ degradation of RhB under visible light within $50 \mathrm{~min}$. With a $k$-value of $0.071 \mathrm{~min}^{-1}, \mathrm{~T}_{-} 650 \mathrm{PP}$ NTs are one of the most efficient visible light photocatalysts to have been reported (see Table S1).

To better explain this improved dye degradation performance of hybrid coaxial NTs under visible light, we first look into the underlying mechanism of photocatalysis of $\mathrm{TiO}_{2}$. Upon light irradiation, electrons are excited from the valence band (VB) of $\mathrm{TiO}_{2}$ to its conduction band (CB), leaving behind holes in its VB and, thus, creating electron-hole pairs on the surface of the semiconductor. ${ }^{[26]}$ Electron-hole pairs are responsible for initiating photocatalytic reactions by converting oxygen and water to form highly reactive species such as $\mathrm{OH}^{\bullet}$ and $\mathrm{O}_{2}{ }^{\circ-}$. These radicals play a crucial role in the photocatalytic degradation of organic molecules like RhB into harmless products (Figure 3d) However, the band gap of $\mathrm{TiO}_{2}$ ranges from $2.98 \mathrm{eV}$ to $3.26 \mathrm{eV}$, depending on its crystalline phase, which limits its light absorption to only the UV region. As can be observed from the UV-Vis diffuse reflectance spectra (DRS) (Figure 3e), the T_650 NTs exhibited a light absorption only within the 250-380 nm range, which can justify their poor photocatalytic activity under visible light (band-gap of $3.02 \mathrm{eV}$ ). Coupling $\mathrm{TiO}_{2}$ with nanosized noble metal structures extends its absorption band into the visible light region, as shown in Figure 3e. Based on the DRS measurement their band-gaps 
can be calculated as $2.82 \mathrm{eV}, 2.84 \mathrm{eV}$ and $2.83 \mathrm{eV}$ for $\mathrm{TP}_{-} 650, \mathrm{~T}_{-650} \mathrm{P}$ and $\mathrm{T}_{-650} \mathrm{PP}$ NTs, respectively. Besides, these noble metal nano structures absorb visible light to generate collective oscillation of valence electrons known as the localized surface plasmon resonance (LSPR). The light excited electrons on the surface of noble metals must overcome the Schottky barrier $(\phi$, difference between the CB of the semiconductor and the work function of metal) created at the interface of semiconductor/metal heterojunction in order to be transferred to the $\mathrm{TiO}_{2}$ phase. ${ }^{[14 \mathrm{~b}, 27]}$ The $\mathrm{CB}$ of $\mathrm{TiO}_{2}$, being an electron acceptor, ${ }^{[28]}$ readily accepts the electrons from $\mathrm{Pd}$ or $\mathrm{PtPd}$, hence promoting diffusion of electrons into the $\mathrm{TiO}_{2}$ shell (Figure 3d). Thus, by coupling noble metals to $\mathrm{TiO}_{2}$ NTs, electron-hole pairs can be efficiently generated under visible light. By comparing Figure $3 \mathrm{c}$ and Figure $3 \mathrm{e}$, we can find that under visible light, the normalized absorbed intensity exhibits a positive correlation with the $k$-values. A higher photocatalytic efficiency is observed in NTs with a continuous interface $\left(\mathrm{T}_{6} 650 \mathrm{P}\right)$ than in samples with randomly distributed NPs (TP_650). Possible explanations for this phenomenon could be that in TP 650 NTs there is a larger availability of $\mathrm{Pd}$ catalyst and an increased interface area between $\mathrm{TiO}_{2}$ and $\mathrm{Pd}$. This can increase the absorption of light and, hence, generate more electron-hole pairs to participate in the photocatalytic degradation process. Another important result obtained shows that the bimetallic NTs $\left(\mathrm{T}_{-} 650 \mathrm{PP}\right)$ are 1.7 times more efficient than the monometallic NTs $\left(\mathrm{T} \_650 \mathrm{P}\right)$. This result can be explained by the fact that combining Pd with Pt lowers its work function, and, therefore, reduces the height of the Schottky barrier. ${ }^{[14 a, 29]}$ A lowered Schottky barrier is able to promote electron transfer from the PtPd NTs to the $\mathrm{TiO}_{2}$ phase much more efficiently, ${ }^{[14 \mathrm{~b}, 30]}$ hence making the bimetallic $\mathrm{T}_{2} 650 \mathrm{PP}$ NTs better photocatalysts.

The improvement in photocatalytic activity under UV light is not as prominent as under visible light. This slightly increased efficiency in $\mathrm{T}_{-650} \mathrm{P}$ and $\mathrm{T} \_650 \mathrm{PP}$ NTs may arise from the fact that noble metals act as electron traps and decrease the electron-hole recombination rate. ${ }^{[9 \mathrm{~b}, 18]}$ A closer look at the graph shows that under UV light, TP_650 NTs 
exhibited a slower degradation rate than T_650 NTs. This trend can be attributed to the presence of many randomly distributed $\mathrm{Pd}$ NPs all over the $\mathrm{TiO}_{2}$ matrix. Such a random NP distribution all over the $\mathrm{TiO}_{2}$ surface can reduce the total photoactive area of $\mathrm{TiO}_{2} \mathrm{NTs}$ and, hence, reduce their photoactivity. ${ }^{[16,31]}$

To develop practical photocatalysts for water purification, it is important that they are not only efficient but also highly stable so that they can be reused. The reusability of coaxial T_650P NTs and NP coupled TP_650 NTs are examined and presented in Figure $3 \mathrm{f}$. In the case of coaxial $\mathrm{T}_{-650} \mathrm{P}$ NTs, all three runs had almost similar and stable degradation rates, expressed by their $k$-values, $0.0425 \mathrm{~min}^{-1}, 0.0425 \mathrm{~min}^{-1}$ and $0.0418 \mathrm{~min}^{-1}$, respectively. TP_650 NTs exhibited a gradually decreased photocatalytic activity with each consecutive run $(0.0244$ $\min ^{-1}, 0.0226 \mathrm{~min}^{-1}$, and $0.0174 \mathrm{~min}^{-1}$ ), respectively. This unstable reusability trend shown by the TP_650 NTs can be caused by the photocorrosion or dissolution of Pd NPs in their surrounding environment over time. ${ }^{[17 a, 17 b]}$ Our results suggest that noble metals should be shielded from the surrounding media by designing core-shell structures instead of using them as NPs, as has been indicated elsewhere. ${ }^{[12 a, 18]}$

Photocatalytic efficiencies of our NTs was also studied under natural sunlight to demonstrate that they can be used for practical water purification applications at a similar [Dye]/[T_650PP] weight ratio of 0.006 (Figure 3g). We can observe almost similar degradation trends as obtained under a visible light lamp. This study shows that our coaxial NTs, especially T_650PP NTs that degraded $100 \%$ of the dye in just $30 \mathrm{~min}$, can serve as efficient photocatalysts to destroy organic pollutants under sunlight. To further demonstrate the practical use of $\mathrm{T}_{-} 650 \mathrm{PP}$ NTs under direct sunlight, we also investigated the photocatalytic degradation of three different organic dyes i.e. $\mathrm{RhB}$, methyl orange (MO) and methylene blue (MB) at much elevated concentrations of $12 \mathrm{mg} \mathrm{L}^{-1}\left([\mathrm{Dy}] /\left[\mathrm{T}_{-} 650 \mathrm{PP}\right]=0.018\right)$. Figure $3 \mathrm{~h}$ presents a collage of images taken every 20 minutes to represent how the $\mathrm{T}_{-} 650 \mathrm{PP}$ NTs were successful in degrading all three dyes in less than $2 \mathrm{~h}$, even at much higher dye concentrations. 
This result further highlights the versatile and efficient nature of water remediation offered by our hybrid nanotubes.

To ensure that these hybrid NTs can be guided to specific locations and efficiently collected for re-use, T_650PP-Ni NTs were tested to study their response in the presence of external fields and/or fuels such as $\mathrm{H}_{2} \mathrm{O}_{2}$ solution. The novel architecture of these coaxial NTs coupled with Ni nanowires, enabled their use under three different propulsion mechanisms, i.e. under wireless magnetic and acoustic fields and as self-propelled catalytic nanomachines (Figure 4a-d). Rotational magnetic fields were applied to a single $\mathrm{T}_{-} 650 \mathrm{PP}-\mathrm{Ni}$ NT released in deionized (DI) water. It was possible to precisely guide the hybrid NT along a pre-planned trajectory, as can be seen from the time lapse image in Figure 4a and Video S1. A single, selfpropelled $\mathrm{T}_{-} 650 \mathrm{PP}-\mathrm{Ni} \mathrm{NT}$ demonstrated autonomous actuation in the presence of $5 \% \mathrm{H}_{2} \mathrm{O}_{2}$ solution. Here, the PtPd NT decomposed the $\mathrm{H}_{2} \mathrm{O}_{2}$ solution into water and oxygen bubbles, giving rise to a self-propelled motion, powered by ejection of oxygen bubbles from the PtPd NT (Figure 4b and Video S2). Figure 4c and Video S3, illustrates dual motion exhibited by a single $\mathrm{T}_{-} 650 \mathrm{PP}-\mathrm{Ni} \mathrm{NT}$ in $\mathrm{H}_{2} \mathrm{O}_{2}$ solution and under wireless magnetic fields. From this timelapse image, it can be clearly seen that static magnetic fields can be used to precisely guide the direction of movement of these autonomous nanomachines. T_650PP-Ni NTs can also be powered wirelessly using acoustic fields as shown in Figure 4d and Video S4, where a single NT displayed an efficient linear propulsion. Hence, by optimal material selection and careful design of our nanomachines, three different locomotion techniques were demonstrated by hybrid T_650PP-Ni NTs. Such versatile actuation exhibited by a single nanomachine can offer a diverse and flexible scope of operation, depending upon its mission or upon changes in its surrounding environment. ${ }^{[23 a]}$ The importance of developing smart photocatalysts that can be easily actuated is further highlighted by comparing the degradation curves obtained under static versus continuous agitation plots presented in Figure S4a and b. From these plots one can see that, without any magnetic actuation our nanotubes displayed much lower 
photocatalytic activity. This could be due to their sedimentation in the absence of any actuation and hence, reduced active surface area availability for the degradation process.

In order to use these hybrid NTs to clean water sources with aquatic life, it is important to ensure that they are biocompatible. Therefore, the interaction between the T_650 PP-Ni NTs and NIH 3 T3 cells was examined for $48 \mathrm{~h}$. Figure 4e shows an optical image of healthy fibroblast cells interacting with the hybrid NTs. To further demonstrate this, we also performed nucleus and actin filament staining which showed viable cells growing over the hybrid NTs (Figure 4f). Additionally, MTT assay was also performed to test the biocompatibility of our hybrid $\mathrm{T}_{-} 650 \mathrm{PP}-\mathrm{Ni}$ NTs after $48 \mathrm{~h}$, and they showed a cell viability of $80 \%$ even when NT concentration was as high as $75 \mathrm{ppm}\left(0.075 \mathrm{mg} \mathrm{mL}^{-1}\right)$ (Figure S5). These results demonstrate that our hybrid NTs are non-cytotoxic.

\section{Conclusions}

In conclusion, we have developed an architecture to create highly efficient visible light photocatalysts for water remediation. These photocatalysts are composed of $\mathrm{TiO}_{2} \mathrm{NTs}$ and noble metals present either as NPs or as coaxial NTs using an electrochemical fabrication approach. We were able to compare for the first time the efficiency of $\mathrm{TiO}_{2} \mathrm{NT}$ s coupled to noble metals as NPs and as NTs. $\mathrm{TiO}_{2}-\mathrm{Pd}$ monometallic NTs, $\mathrm{TiO}_{2}-\mathrm{PtPd}$ bimetallic NTs and $\mathrm{TiO}_{2}$ NTs decorated with Pd NPs were fabricated. We observed that coaxial $\mathrm{TiO}_{2}-\mathrm{Pd}$ NTs exhibited a much higher efficiency and stability in comparison to $\mathrm{TiO}_{2} \mathrm{NT}$ s covered with $\mathrm{Pd}$ NPs. Furthermore, when $\mathrm{TiO}_{2}$ NTs coupled with bimetallic PtPd NTs were used, they demonstrated a much faster degradation rate in comparison to $\mathrm{TiO}_{2} \mathrm{NTs}$ coupled with monometallic Pd NTs. The most efficient photocatalysts, with a $k$-value of $0.071 \mathrm{~min}^{-1}$, were the $\mathrm{TiO}_{2}-\mathrm{PtPd}$ coaxial NTs, which were able to degrade the organic pollutant $\mathrm{RhB}$, in situ, with a $100 \%$ efficiency in $50 \mathrm{~min}$ and $30 \mathrm{~min}$ under visible light and natural sunlight, respectively. The unique design of hybrid $\mathrm{TiO}_{2}-\mathrm{PtPd}-\mathrm{Ni} \mathrm{NTs}$, apart from their enhanced 
photocatalytic performance, also provided the advantage of optimally harvesting both fuelfree and fuel-powered actuations. Such versatile propulsion mechanisms make these hybrid nanomachines cost-effective and highly efficient photocatalysts that can be precisely controlled and collected for re-use. Biocompatibility demonstrated by these nanomachines further confirms their possible utilization for water purification with aquatic life. The hybrid nanomachines developed in this work are attractive candidates for designing future water remediation and water splitting photocatalysts and display efficient motion under complex, challenging environments.

\section{Experimental section}

Fabrication of $\mathrm{TiO}_{2}$ nanotubes: Commercially available AAO templates with a pore diameter of $200 \mathrm{~nm}$ were purchased from Whatman Anodisc. $100 \mathrm{~nm}$ Au was evaporated by electron beam evaporation on one side of the template that served as a conductive working electrode. Prior to deposition, the Au covered side of the template was isolated to ensure exclusive deposition inside the pores. Electrodeposition was carried out using an Autolab PGSTAT302N, in a three-electrode setup with Pt sheet as a counter electrode and a $\mathrm{Ag} / \mathrm{AgCl}$ $(3 \mathrm{M} \mathrm{KCl})$ reference electrode. $\mathrm{TiO}_{2}$ nanotubes were fabricated at room temperature from an electrolyte solution consisting of $0.02 \mathrm{M}$ titanium oxysulfate $\left(\mathrm{TiOSO}_{4}\right), 0.03 \mathrm{M}$ hydrogen peroxide $\left(\mathrm{H}_{2} \mathrm{O}_{2}\right), 0.05 \mathrm{M} \mathrm{HNO}_{3}$ and $0.25 \mathrm{M} \mathrm{KNO}_{3}$ at a $\mathrm{pH}$ of $1.4 .{ }^{[16,24]}$ Deposition was carried out potentiostatically at a constant voltage of $-1.00 \mathrm{~V}$ for $6 \mathrm{~h}$. After deposition, the AAO templates were either dried overnight at $100^{\circ} \mathrm{C}$ or annealed for $2 \mathrm{~h}$ at $650^{\circ} \mathrm{C}$ in a tube furnace.

Fabrication of Pd, Pt-Pd nanotubes: For Pd and Pt-Pd NT fabrication, a three-electrode setup was used with the AAO template filled with $\mathrm{TiO}_{2}$ nanotubes serving as the working electrode, a Pt sheet acting as the counter electrode and a $\mathrm{Ag} / \mathrm{AgCl}(3 \mathrm{M} \mathrm{KCl})$ reference electrode. Pd electrodepostion was carried out potentiostatically at room temperature under 
constant magnetic stirring at a potential of $-0.1 \mathrm{~V}$ for 30 min inside $\mathrm{T}_{-} 650 \mathrm{NTs}$ and for $10 \mathrm{~min}$ inside $\mathrm{T}_{-} 100 \mathrm{NTs}$. Plating solution consisted of $0.05 \mathrm{M} \mathrm{PdCl}_{2}$ and $0.1 \mathrm{M} \mathrm{HCl}^{[32]} \mathrm{Pt}-\mathrm{Pd}$ bimetallic nanotubes were fabricated using the same setup as above at a potential of $-0.1 \mathrm{~V}$ for $3 \mathrm{~h}$ with a plating solution consisting of $0.005 \mathrm{M} \mathrm{PdCl}_{2}, 0.005 \mathrm{M} \mathrm{H}_{2} \mathrm{PtCl}_{6}$ and $0.04 \mathrm{M} \mathrm{HCl}^{[33]}$

Fabrication of Ni nanowires: For Ni nanowire fabrication, a similar three-electrode set-up was used with the AAO template filled with $\mathrm{TiO}_{2}$ and $\mathrm{Pd}$ or Pt-Pd nanotubes serving as the working electrode, a Pt sheet acting as the counter electrode and a $\mathrm{Ag} / \mathrm{AgCl}\left(0.1 \mathrm{M} \mathrm{Na}_{2} \mathrm{SO}_{4}\right)$ reference electrode. Nickel electrodepostion was carried out potentiostatically at room temperature under constant magnetic stirring at a potential of $-1.0 \mathrm{~V}$ for $2 \mathrm{~min}$, using a plating solution consisting of $0.4 \mathrm{M} \mathrm{NiSO}_{4}$ and $0.7 \mathrm{M}$ boric acid. After deposition, the nanotubes were released by dissolving the AAO template in an aqueous solution of $5 \mathrm{M} \mathrm{NaOH}$ for $2 \mathrm{~h}$ followed by washing them with DI water five times using ultrasonification. Finally, the nanotubes were collected using centrifugation and stored in DI water.

Material characterization: Morphology of the resulting nanotubes was studied by scanning electron microscopy (SEM, Zeiss ULTRA 55, Zeiss, Oberkochen, Germany), transmission electron microscopy (TEM, FEI F30, FEI Co., Hillsboro, OR), and scanning transmission electron microscopy (STEM, FEI F30). The composition and crystallographic structure of the nanotubes were analyzed by energy-dispersive X-ray analysis (EDX) and X-ray diffraction (XRD, Bruker AXS D8 Advance). Distribution of elements along the nanotubes was studied by EDX mapping using HAADF STEM (FEI Talos F200X). Local crystallographic structure was studied by selected area electron diffraction (SAED). UV-Vis diffuse reflectance spectra (DRS) was acquired by a Cary $4000 \mathrm{UV}$-vis spectrophotometer. $\mathrm{BaSO}_{4}$ was used as the reflectance standard and the ratio of our sample to $\mathrm{BaSO}_{4}$ was kept constant with respect to the quantity of $\mathrm{TiO}_{2}$. 
Photocatalytic characterization: Photocatalytic experiments were performed at ambient temperature and pressure to study the degradation of $\mathrm{RhB}$ dye in the presence of our nanotube structures after the adsorption-desorption equilibrium was reached. An RhB concentration of $4 \mathrm{mg} \mathrm{L}^{-1}$ was chosen to perform degradation experiments under continuous magnetic agitation. $10 \mathrm{mg}$ of released pure $\mathrm{TiO}_{2}$ and $20 \mathrm{mg}$ of hybrid nanotubes (one whole electroplated AAO template in each case) were dispersed in $30 \mathrm{~mL}$ of $\mathrm{RhB}$ solution and were magnetically stirred. The samples were irradiated with UV-visible light using a $450 \mathrm{~W}$ Xenon lamp (Oriel, model 66929: $450 \mathrm{~W}$ ), where the distance between the lamp and the samples was fixed to $15 \mathrm{~cm}$. Visible light illumination $(\lambda>420 \mathrm{~nm})$ was provided by a colored glass filter (Newport) and UV light illumination was obtained by using a filter with $\lambda<400 \mathrm{~nm}$ (Edmund Optics). A UV-Vis spectrophotometer (Tecan Infinite 200 Pro) was used to obtain the fluorescent spectra of $\mathrm{RhB}$ over time by taking aliquots of irradiated $\mathrm{RhB}$ solution every 10 minutes $(50 \mu \mathrm{L})$ for 1 h.

Manipulation experiments: Hybrid T_650PP-Ni nanotube structures were used for manipulation experiments. The magnetic manipulation system consists of eight stationary electromagnets with soft magnetic cores, capable of producing magnetic fields and gradients up to $50 \mathrm{mT}$ and $0.5 \mathrm{~T} \mathrm{~m}^{-1}$ at frequencies up to $100 \mathrm{~Hz}$. The system is integrated with an inverted fluorescence microscope (Olympus IX 81) and videos of manipulated structures were recorded under rotational and static magnetic fields at 40x magnification (Videos S1 and S3). Manipulation experiments in $\mathrm{H}_{2} \mathrm{O}_{2}$ were performed by adding $5 \% \mathrm{H}_{2} \mathrm{O}_{2}$ solution to the T_650PP-Ni NTs and videos of NTs were recorded at 40x (Videos S2 and S3). Manipulation of hybrid T_650PP-Ni NTs was perfomed by studying their propulsion in a complex standing wave field. A standing wave field was formed by introducing acoustic waves via a piezoelectric transducer bonded on a glass slide adjacent to the liquid droplet. The droplet was 
then sandwiched between glass slides. Acoustic fields at a frequency of $366 \mathrm{kHz}$ and output amplitude of $10 \mathrm{Vp}-\mathrm{p}$ were used to propel $\mathrm{T}_{-650} \mathrm{PP}$ nanotubes (Video $\mathrm{S} 4$, 40x magnification).

Biocompatibility test: NIH 3T3 cells (American Type Cell Culture, ATCC) were expanded in high glucose (4.5 $\left.\mathrm{g} \mathrm{L}^{-1}\right)$ DMEM containing (GIBCO), 10\% fetal calf serum and 100 units $\mathrm{mL}^{-}$ ${ }^{1}$ Penicillin and $100 \mathrm{mg} \mathrm{mL}^{-1}$ Streptomycin. The cells were labeled with Hoechst 33342 (Sigma) and Alexa 488 Phalloidin (Sigma). Phase-contrast and fluorescence images were taken with an IX 81 Olympus camera at 20x magnification. The MTT Cytotoxicity study was conducted in a flat bottomed 96-well tissue culture plate with $4 \times 10^{3} 3 \mathrm{~T} 3$ cells in $100 \mu \mathrm{L}$ culture medium (DMEM, 10\% FCS, 1× Antimycoticum) per well. Cells were allowed to attach on the surface for 5 hours. Then the cell culture medium was supplemented with the hybrid nanotubes at different concentrations $(0-75 \mathrm{ppm})$. After $48 \mathrm{~h}$ of incubation at $37{ }^{\circ} \mathrm{C}$ and $5 \% \mathrm{CO}_{2}, 10 \mu \mathrm{L}$ of $12 \mathrm{mM}$ MTT were added. Cells were further incubated for $4 \mathrm{~h}$ for MTT cleavage at $37{ }^{\circ} \mathrm{C}$. Then, $100 \mu \mathrm{L}$ of isopropanol and $0.04 \mathrm{M} \mathrm{HCl}$ were added to each well and mixed thoroughly until the formazan crystals were dissolved. Absorbance measurements were conducted in a micro-titer plate reader (Infinite F200 Tecan) at $570 \mathrm{~nm}$. Reported values are represented as mean values of five independent replicates as percentages with respect to the control sample (Figure S5).

\section{Supporting Information}

\section{Acknowledgements}

This work has been financed by the European Research Council Starting Grant "Magnetoelectric Chemonanorobotics for Chemical and Biomedical Applications (ELECTROCHEMBOTS)", by the ERC grant agreement no. 336456. The authors would like 
to acknowledge the Scientic Center for Optical and Electron Microscopy (ScopeM) of ETH Zurich, the Institute of Geochemistry and Petrology and the FIRST laboratory, ETH Zurich for their technical support. The authors would also like to thank Kakeru Fujiwara from ETH Zurich for providing the UV-Vis lamp set-up, Dr. Alla Sologubenko from ETH Zurich for help with EDX mappings. The authors would like to acknowldge Dr. Davide Ferri from the Paul Scherrer Institute (PSI) for help with DRS measurements and Dr. Eva Pellicer from Universitat Autònoma de Barcelona for all her support.

Received: ((will be filled in by the editorial staff))

Revised: ((will be filled in by the editorial staff)) Published online: ((will be filled in by the editorial staff))

[1] A. Matilainen, M. Vepsäläinen, M. Sillanpää, Adv. Colloid Interface Sci. 2010, 159, 189.

[2] a) R. Kant, J. Nat. Sci. 2012, 4, 22; b) M. A. Fox, M. T. Dulay, Chem. Rev. 1993, 93, 341.

[3] a) B. Padhi, Int. J. Env. Sci. 2012, 3, 940; b) F. Chequer, G. Oliveira, J. C. E. Ferraz, M. Zanoni and D. Oliveira, Eco Friendly Textile Dyeing and Finishing, InTech, 2013.

[4] P. V. Kamat, D. Meisel, C. R. Chim. 2003, 6, 999.

[5] E. M. Saggioro, A. S. Oliveira, T. Pavesi, C. G. Maia, L. F. V. Ferreira, J. C. Moreira, Molecules 2011, 16, 10370.

[6] D. Mohan, C. U. Pittman Jr, J. Hazard Mater. 2007, 142, 1.

[7] a) S. Malato, P. Fernández-Ibáñez, M. I. Maldonado, J. Blanco, W. Gernjak, Catal.

Today 2009, 147, 1; b) U. I. Gaya, A. H. Abdullah, J. Photochem. and Photobiol. C:

Photochem. Rev. 2008, 9, 1.

[8] a) H. S. M. Tabaei, M. Kazemeini, M. Fattahi, Scientia Iranica 2012, 19, 1626; b) R. Singh, R. Bapat, L. Qin, H. Feng, V. Polshettiwar, ACS Catal. 2016, 6, 2770. 
[9] a) J. Tian, Y. Sang, G. Yu, H. Jiang, X. Mu, H. Liu, Adv. Mater. 2013, 25, 5074; b) S. Sakthivel, M. V. Shankar, M. Palanichamy, B. Arabindoo, D. W. Bahnemann, V. Murugesan, Water Res. 2004, 38, 3001.

[10] R. Ren, Z. Wen, S. Cui, Y. Hou, X. Guo, J. Chen, Sci. Rep. 2015, 5, 10714.

[11] a) H. Zeng, J. Xie, H. Xie, B.-L. Su, M. Wang, H. Ping, W. Wang, H. Wang, Z. Fu, J. Mater. Chem. A 2015, 3, 19588; b) J. Senthilnathan, L. Philip, J. Chem. Eng. 2010, 161, 83; c) Y. Yang, P. Wang, Polymer 2006, 47, 2683; d) M. Wang, L. Sun, Z. Lin, J. Cai, K. Xie, C. Lin, Energy Environ. Sci. 2013, 6, 1211.

[12] a) N. Zhang, S. Liu, X. Fu, Y.-J. Xu, J. Phys. Chem. C 2011, 115, 9136; b) I. Paramasivam, J. M. Macak, P. Schmuki, Electrochem. commun. 2008, 10, 71.

[13] a) R. van de Krol, Y. Liang, J. Schoonman, J. Mater. Chem. 2008, 18, 2311; b) J. Ma, M. Yang, Y. Sun, C. Li, Q. Li, F. Gao, F. Yu, J. Chen, Physica E: Low Dimens. Syst. Nanostruct. 2014, 58, 24.

[14] a) A. Zielińska-Jurek, J. Hupka, Catal. Today 2014, 230, 181; b) Y. Shiraishi, H.

Sakamoto, Y. Sugano, S. Ichikawa, T. Hirai, ACS Nano 2013, 7, 9287; c) J. He, I. Ichinose, T. Kunitake, A. Nakao, Y. Shiraishi, N. Toshima, J. Am. Chem. Soc. 2003, 125, 11034.

[15] a) W. J. Youngblood, S.-H. A. Lee, K. Maeda, T. E. Mallouk, Acc. Chem. Res. 2009, 42, 1966; b) C. Liu, J. Tang, H. M. Chen, B. Liu, P. Yang, Nano Lett. 2013, 13, 2989.

[16] A. W. Maijenburg, J. Veerbeek, R. de Putter, S. A. Veldhuis, M. G. C. Zoontjes, G. Mul, J. M. Montero-Moreno, K. Nielsch, H. Schafer, M. Steinhart, J. E. ten Elshof, J. Mater. Chem. A 2014, 2, 2648.

[17] a) X.-F. Wu, H.-Y. Song, J.-M. Yoon, Y.-T. Yu, Y.-F. Chen, Langmuir 2009, 25, 6438; b) X.-F. Wu, Y.-F. Chen, J.-M. Yoon, Y.-T. Yu, Mater. Lett. 2010, 64, 2208; c) C. Fang, H. Jia, S. Chang, Q. Ruan, P. Wang, T. Chen, J. Wang, Energy Environ. Sci. 2014, 7, 3431.

[18] X. Zhang, S. Ye, X. Zhang, L. Wu, J. Mater. Chem. C 2015, 3, 2282. 
[19] Q. Dong, H. Yu, Z. Jiao, G. Lu, Y. Bi, RSC Adv. 2014, 4, 59114.

[20] a) T. Y. Seiichi Sawatani, Tomokazu Ohya, Takayuki Ban, Yasutaka Takahashi and Hideki Minoura, Electrochem. Solid State Lett. 2005, 8, C69; b) C. N. a. G. Nogami, J. Electrochem. Soc. 1996, 143, 1547.

[21] F. Mushtaq, M. Guerrero, M. S. Sakar, M. Hoop, A. M. Lindo, J. Sort, X. Chen, B. J. Nelson, E. Pellicer, S. Pane, J. Mater. Chem. A 2015, 3, 23670.

[22] a) W. Gao, S. Sattayasamitsathit, J. Orozco, J. Wang, J. Am. Chem. Soc. 2011, 133, 11862; b) X.-Z. Chen, N. Shamsudhin, M. Hoop, R. Pieters, E. Siringil, M. S. Sakar, B. J. Nelson, S. Pane, Mater. Horiz. 2016, 3, 113; c) J. Li, V. V. Singh, S. Sattayasamitsathit, J. Orozco, K. Kaufmann, R. Dong, W. Gao, B. Jurado-Sanchez, Y. Fedorak, J. Wang, ACS Nano 2014, 8, 11118.

[23] a) J. Li, T. Li, T. Xu, M. Kiristi, W. Liu, Z. Wu, J. Wang, Nano Lett. 2015, 15, 4814; b) V. Garcia-Gradilla, J. Orozco, S. Sattayasamitsathit, F. Soto, F. Kuralay, A. Pourazary, A. Katzenberg, W. Gao, Y. Shen, J. Wang, ACS Nano 2013, 7, 9232.

[24] Z. Miao, D. Xu, J. Ouyang, G. Guo, X. Zhao, Y. Tang, Nano Lett. 2002, 2, 717.

[25] a) A. Di Paola, G. Cufalo, M. Addamo, M. Bellardita, R. Campostrini, M. Ischia, R. Ceccato, L. Palmisano, Colloids Surf. A Physicochem. Eng. Asp. 2008, 317, 366; b) R. Li, Y. Weng, X. Zhou, X. Wang, Y. Mi, R. Chong, H. Han, C. Li, Energy Environ. Sci. 2015, 8, 2377.

[26] a) B. A. Wols, C. H. M. Hofman-Caris, Water Res. 2012, 46, 2815; b) V. I. Parvulescu, H. Garcia, in Catalysis. 2011, 23, 204.

[27] a) S. Linic, P. Christopher, D. B. Ingram, Nat. Mater. 2011, 10, 911; b) Y. Nakato, K. Ueda, H. Yano, H. Tsubomura, J. Phys. Chem. 1988, 92, 2316.

[28] K. H. Leong, H. Y. Chu, S. Ibrahim, P. Saravanan, Beilstein J. Nanotechnol. 2015, 6, 428.

[29] A. Zielinska-Jurek, J. Nanomater. 2014, 2014, 17. 
[30] A. G. Dylla, K. J. Stevenson, Chem. Commun. 2011, 47, 12104.

[31] F. B. Li, X. Z. Li, Chemosphere 2002, 48, 1103.

[32] L. Liu, S. Park, Chem. Mater. 2011, 23, 1456.

[33] S. M. Kim, L. Liu, S. H. Cho, H. Y. Jang, S. Park, J. Mater. Chem. A 2013, 1, 15252. 
(a)
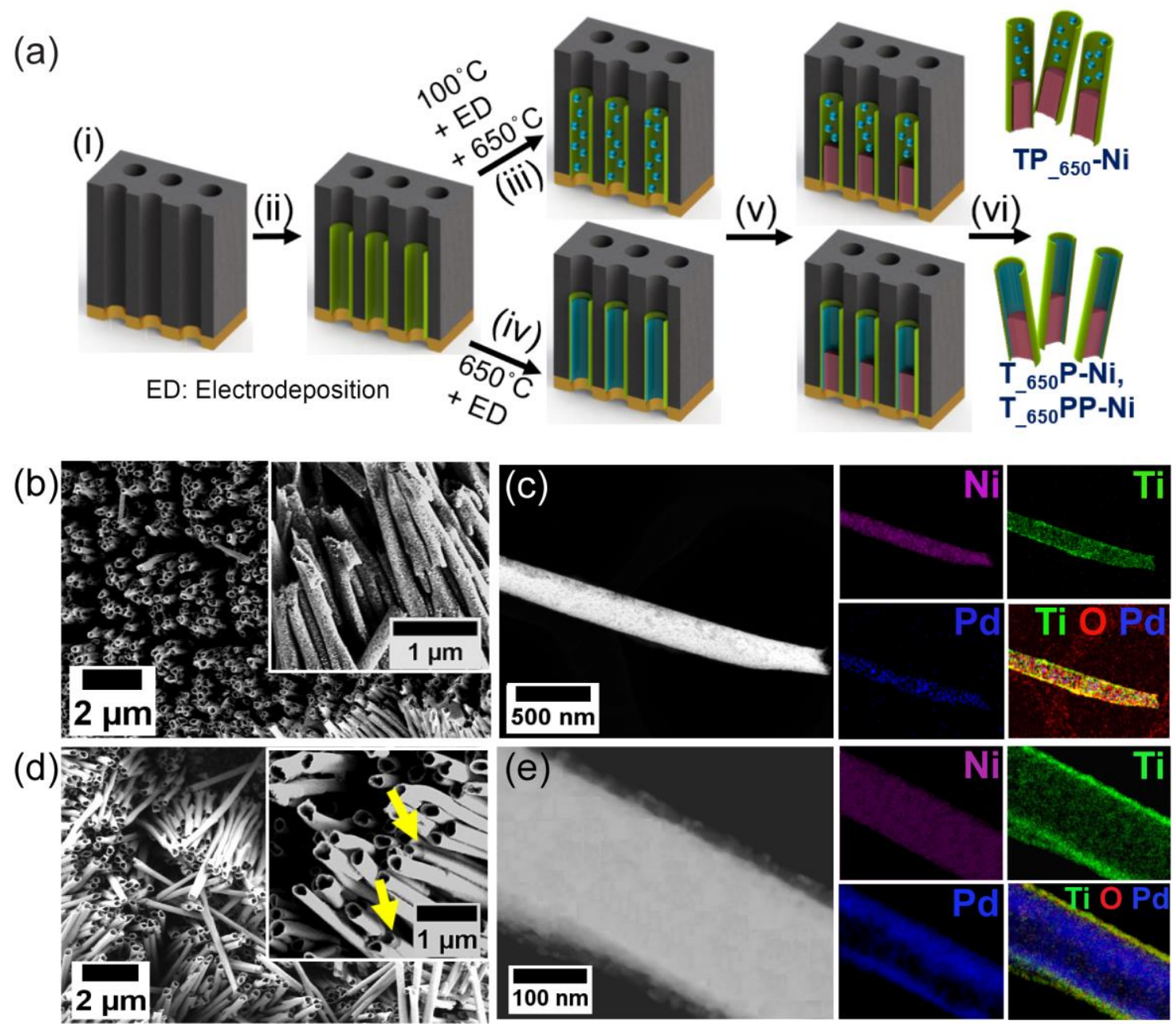

Figure 1. Fabrication overview of hybrid NTs: (a) (i) Au sputtered on one side of AAO templates, (ii) electrochemically induced sol-gel deposition of $\mathrm{TiO}_{2}$ NTs, (iii) electrodeposition (ED) of $\mathrm{Pd} \mathrm{NPs}$ inside $\mathrm{TiO}_{2} \mathrm{NTs}$ dried at $100{ }^{\circ} \mathrm{C}$, followed by annealing at $650{ }^{\circ} \mathrm{C}$, (iv) electrodeposition of $\mathrm{Pd} \mathrm{NTs}$ inside $\mathrm{TiO}_{2} \mathrm{NTs}$ annealed at $650{ }^{\circ} \mathrm{C}$, (v) electrodeposition of Ni NWs, followed by (vi) etching of AAO templates. (b) SEM image showing an overview of freestanding $\mathrm{TP}_{-650}$ NTs and the inset shows its magnified image with presence of NPs distributed on the $\mathrm{TiO}_{2}$ surface. (c) HAADF STEM image of a single TP $650-\mathrm{Ni}$ NT and its corresponding EDX Maps obtained for Ni, Ti, Pd and a superimposed image that clearly shows the presence of Pd as NPs inside a $\mathrm{TiO}_{2}$ NT. (d) SEM image showing an overview of freestanding $\mathrm{T}_{-} 650 \mathrm{P}$ NTs and the inset shows its magnified image. (e) HAADF STEM image of a single $\mathrm{T}_{-650} \mathrm{P}-\mathrm{Ni}$ NT and its corresponding EDX maps obtained for $\mathrm{Ni}, \mathrm{Ti}, \mathrm{Pd}$ where the superimposed image clearly shows a coaxial $\mathrm{TiO}_{2}-\mathrm{Pd} \mathrm{NT}$ structure. 
(a)

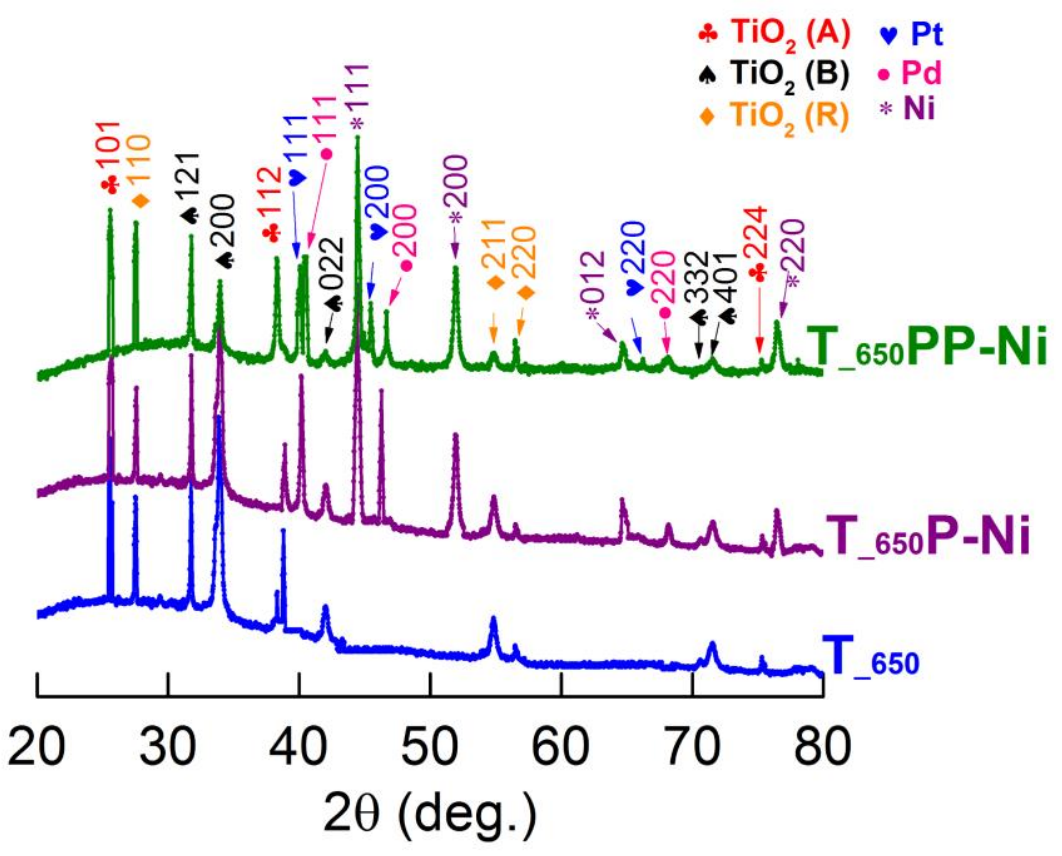

(b)
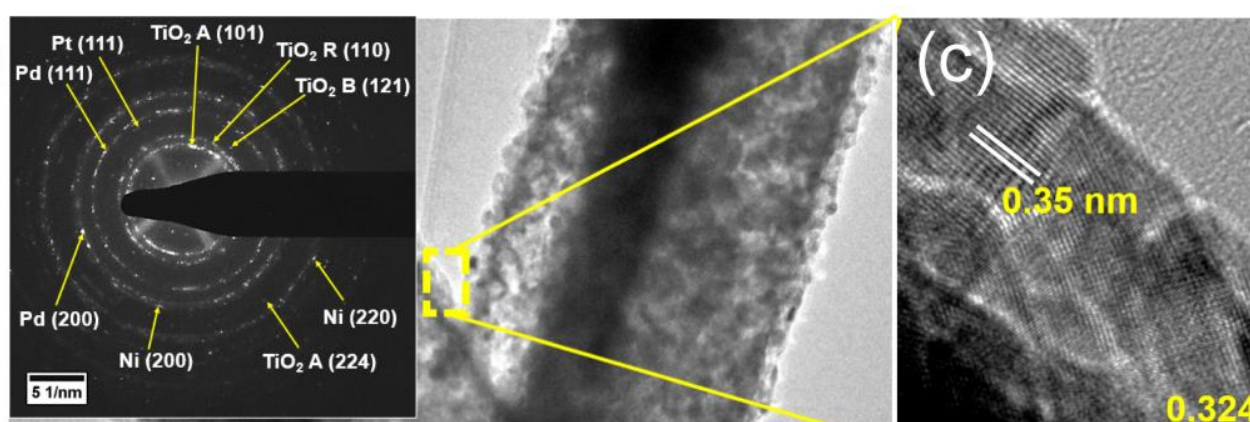

$3 \mathrm{~nm}$

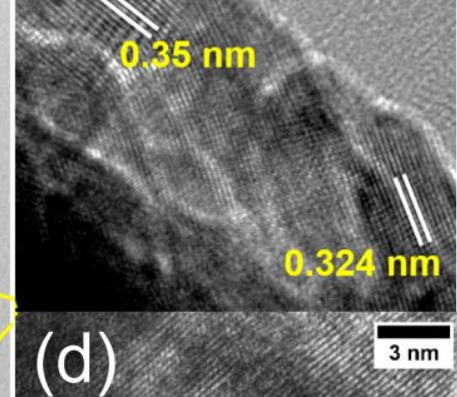

$\mathrm{TiO}_{2} \mathrm{NT}$
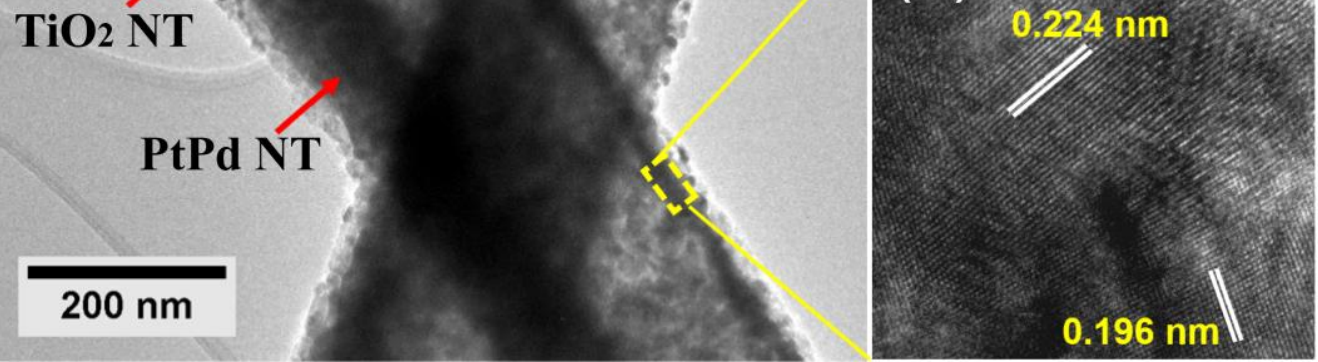

Figure 2. Structural characterisation of hybrid NTs. (a) XRD patterns obtained for hybrid $\mathrm{TiO}_{2}$ NTs. (b) TEM image showing two overlapping $\mathrm{T}_{-} 650 \mathrm{PP}-\mathrm{Ni}$ NTs. In the inset, its corresponding diffraction pattern can be observed. (c) HRTEM image obtained from the outermost shell showing the presence of anatase and rutile $\mathrm{TiO}_{2}$ whereas, (d) the HRTEM image obtained from the interface region shows the presence of metallic Pt and Pd. 
(a)

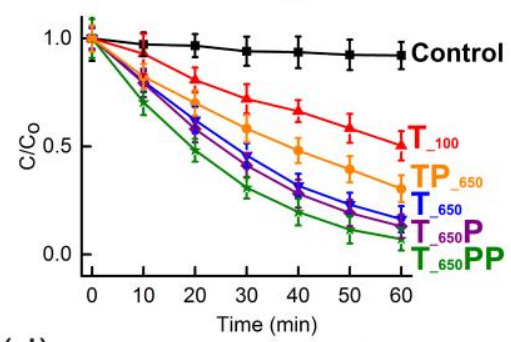

(d)

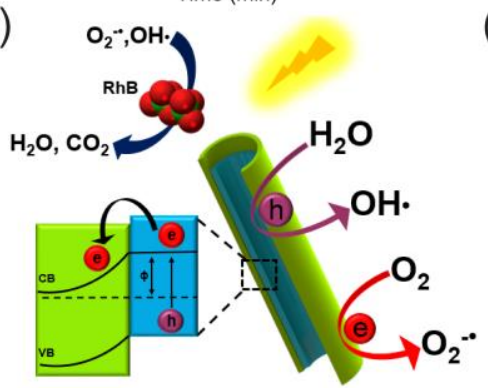

(g)

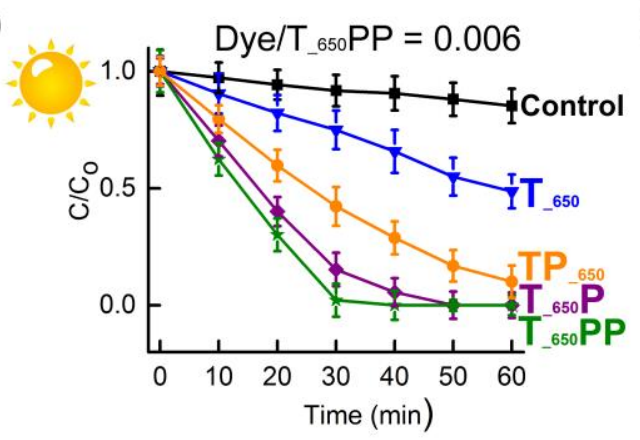

(b) Visible Light

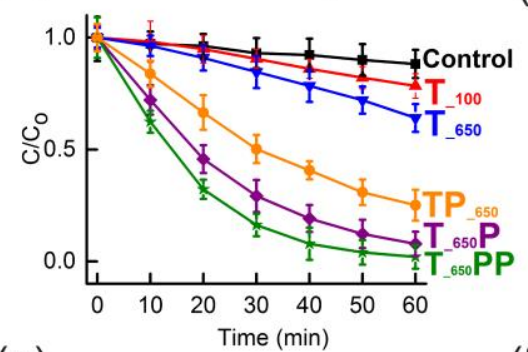

(e)

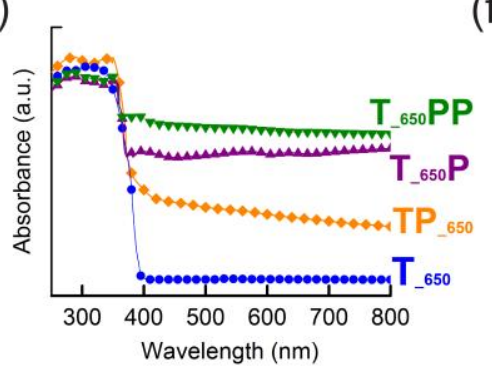

(c)

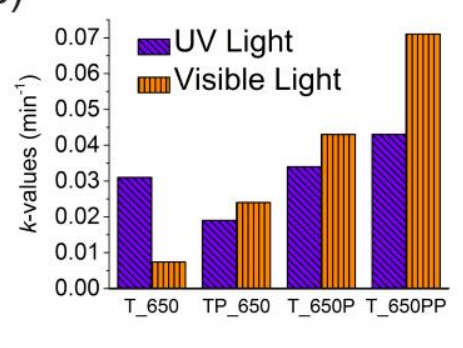

(f)

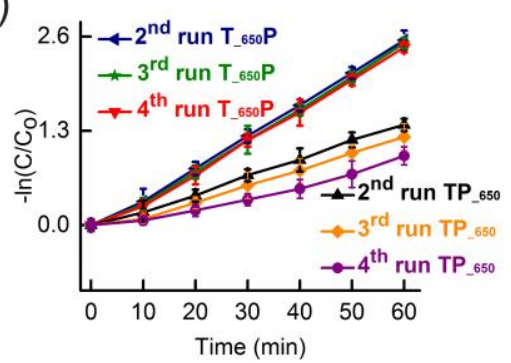

(h)

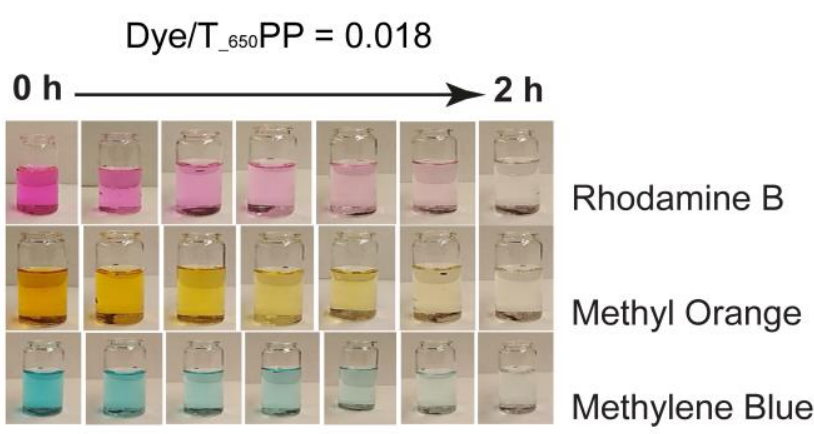

Figure 3. Photocatalytic degradation of RhB dye using hybrid NTs. Photocatalytic degradation curves obtained for $\mathrm{RhB}$ under (a) UV light irradiation and (b) visible light irradiation. (c) Comparison of degradation rate constants under UV and visible light for RhB. (d) Photocatalytic degradation scheme of hybrid coaxial NTs under visible light showing the formation of electron-hole pairs and how they degrade organic pollutants. (e) UV-Vis DRS spectra obtained for $\mathrm{TiO}_{2}$ NTs with and without noble metals. (f) Linear fit log plots of three consecutive degradation curves showing the reusability trend exhibited by TP_650 NTs and T_650P NTs. (g) Photocatalytic degradation curves obtained under natural sunlight, where RhB concentration was $4 \mathrm{mg} \mathrm{L}^{-1}$ and (h) visual color change of three different organic dyes at a higher concentration of $12 \mathrm{mg} \mathrm{L}^{-1}$ under natural sunlight over time. 

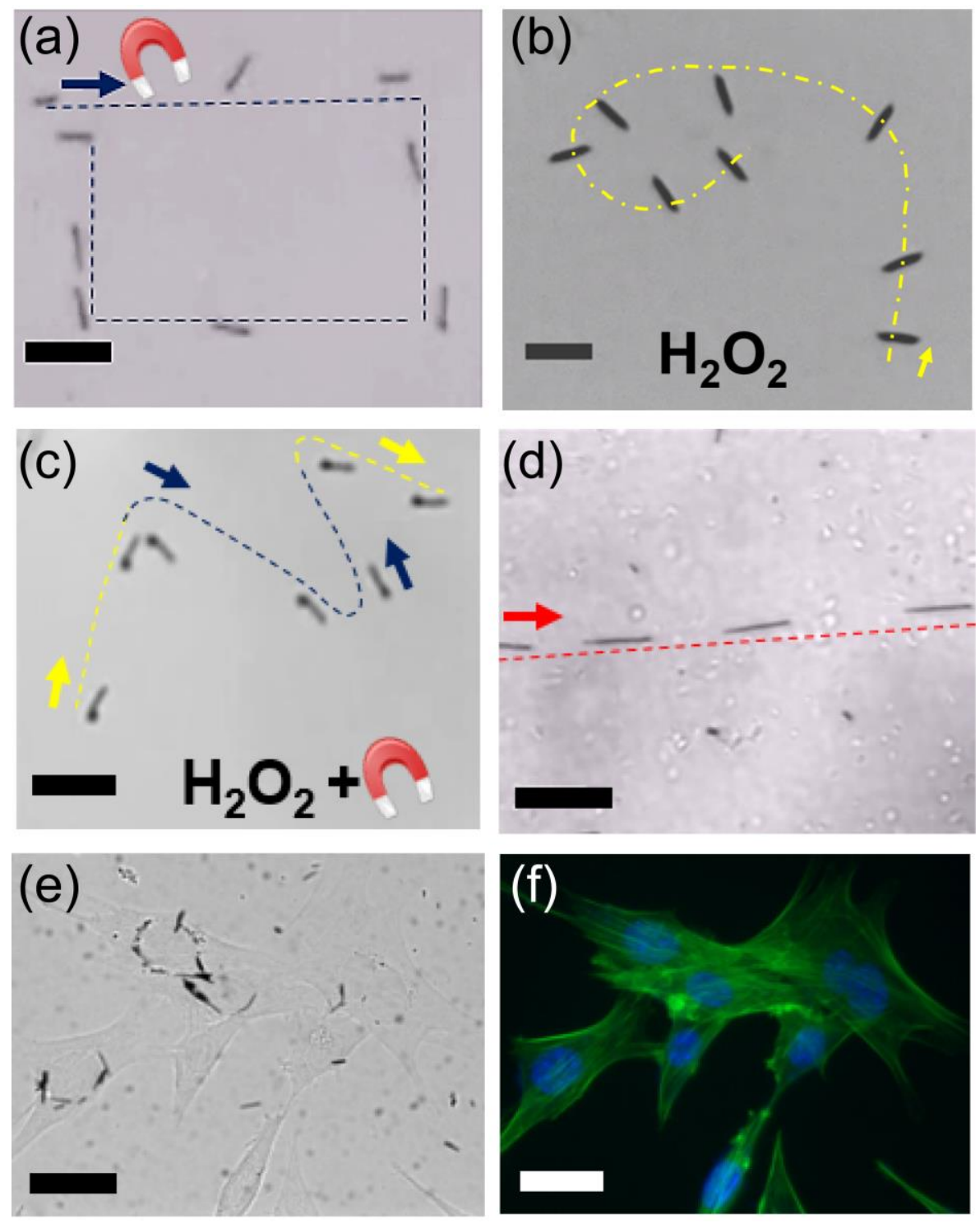

Figure 4. Propulsion mechanisms and biocompatibilty of hybrid $\mathrm{TiO}_{2}-\mathrm{PtPd}-\mathrm{Ni}$ NTs. (a) Time lapse image showing magnetic manipulation of a hybrid NT along a pre-planned trajectory (blue); (b) Time lapse image showing an autonomous motion demonstrated by a single hybrid NT in $5 \% \mathrm{H}_{2} \mathrm{O}_{2}$ solution (yellow); (c) time lapse image of a hybrid NT showing dual propulsion exhibiting an autonmous motion (yellow) which can also be guided by wireless magnetic fields (blue). (e) Optical image of NIH $3 \mathrm{~T} 3$ fibroblast cells cultured on $\mathrm{TiO}_{2}-\mathrm{PtPd}-$ Ni NTs showing cell proliferation; and (f) its corresponding fluorescence image with stained nucleus (blue) and actin (green) showing healthy cells with extended actin fibers. Scale bars indicate $30 \mu \mathrm{m}(\mathrm{a}-\mathrm{c}), 40 \mu \mathrm{m}(\mathrm{d}), 80 \mu \mathrm{m}(\mathrm{e}, \mathrm{f})$. 
Coaxial $\mathrm{TiO}_{2}$-PtPd-Ni hybrid nanotubes exhibit greatly enhanced visible light photocatalyic activity: a 100\% degradation of organic pollutant under visible and natural sunlight with high stability. Combined with multiple locomotion strategies, these highly efficient and cost-effective hybrid photocatalytic nanomachines have the potential to be reused for practical water remediation applications in complex and challenging environments.

Keywords: $\mathrm{TiO}_{2}$; Visible light photocatalysis; Core-shell nanotubes; Electrochemical synthesis; Micromanipulation

Authors: F. Mushtaq, A. Asani, M. Hoop, Dr. X.-Z. Chen*, Dr. D. Ahmed, Prof. Dr. B. J. Nelson, Dr. S. Pané*

Multi-Scale Robotics Lab (MSRL), Institute of Robotics and Intelligent Systems (IRIS), ETH Zurich, CH-8092 Zurich, Switzerland.

Title: Highly Efficient Coaxial TiO2-PtPd Tubular Nanomachines for Photocatalytic Water Purification with Multiple Locomotion Strategies

TOC Figure:

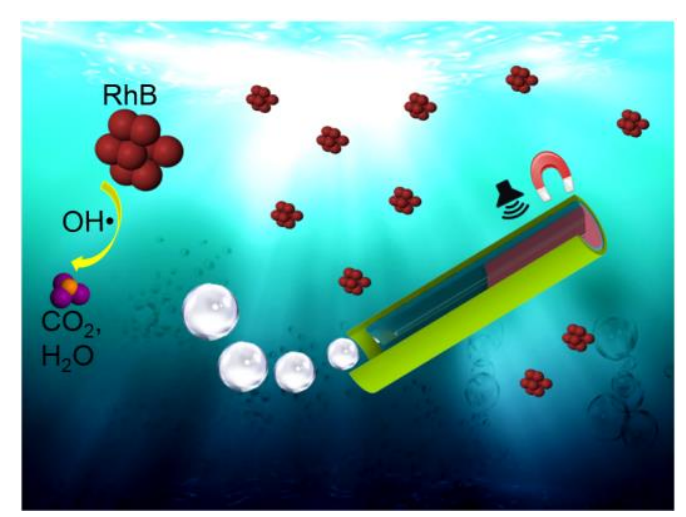

